\title{
Design and simulation of metamorphic lower limbs rehabilitation robot with two gaits modes
}

\begin{abstract}
The walking gait trajectory curve of normal people is given. The stepping gait trajectory curve of normal people is obtained by taking videos of the tester's stepping up process and then getting the coordinates of marked points with MATLAB. The Metamorphic Mechanism and Mechanical structure of pedal lower limbs rehabilitation training robot with walking gait and stepping gait are designed. Based on ADAMS, kinematics simulation and analysis of walking gait mechanism and stepping gait mechanism of lower limbs rehabilitation robot are carried out, which verifies that the walking gait mechanism and stepping gait mechanism of the metamorphic lower limbs rehabilitation robot can realize the walking gait trajectory curve and stepping gait trajectory curve of normal people.
\end{abstract}

Keywords: lower limbs rehabilitation robot, metamorphic mechanism, walking gait mechanism, step gait mechanism
Volume 5 Issue 3 - 2019

\author{
jirong Wang,' Yuhang Zheng,' Yanrui Bi,' \\ Wenlong Wang, ${ }^{2}$ Jun $\mathrm{Li}^{2}$ \\ 'College of Mechanical and Electronic Engineering, Qingdao \\ University, China \\ ${ }^{2}$ College of computer science and technology, Qingdao \\ University, China
}

Correspondence: Jun Li, College of computer science and technology, Qingdao University, China, Tel I370899892I, Email lijun@qdu.edu.cn

Received: April 16, 2019 | Published: May 28, 2019

\section{Introduction}

Rehabilitation robot is a kind of training equipment, which can be used in many places including rehabilitation training at home. The lower limbs rehabilitation robot is a kind of lower limbs rehabilitation aid, which plays an important role in the rehabilitation treatment of cerebral palsy, hemiplegia, paraplegia and other paralytic diseases. Through the use of lower limbs rehabilitation robots, the abnormal postures of patients can be corrected, gait and walking ability can be improved, and then self-care ability of life can be improved, so that people with lower limbs movement disorders can gradually live independently and their quality of life can be significantly improved. A lower limbs rehabilitation training robot with pedal driven by planetary gear train, Gait Trainer (GIT), is designed by Henning Schmidt of the Franhofer IPK and Stefan Hessen of Charite University Hospital, etc., which can imitate the foot posture of normal people when they are walking, so as to assist the rehabilitation trainee to complete the rehabilitation training action. On the basis of GIT, its second generation has been developed by Haptic Walker, which can assist the trainees to complete various rehabilitation training, such as walking on the ground, pedaling bicycles, Stepping up the stairs and skiing. ${ }^{1}$

Healthsouth Corporation of the United States has developed a rehabilitation robot called "Autoambulator", which is a two-degreeof-freedom manipulator and controls two sets of motors to achieve lower limbs traction, thus realizing the gait rehabilitation training. Autoambulator can monitor all kinds of data of patients during rehabilitation training, and can make accurate evaluations of patients' training status at any time. ${ }^{2,3}$

At the same time, other research institutes have also done some relevant researches. Fraunhofer IPK has designed a rope-driven rehabilitation training robot; ${ }^{4}$ Makinkawa Laboratory in Japan has designed a rehabilitation robot of lower limbss which can simulate various human movements, and the robot can achieve rehabilitation training such as stepping up and down stairs; ${ }^{5}$ Free University of Berlin in Germany has designed a MGT-type rehabilitation robot. ${ }^{6}$ The University of Salford, UK, has designed a dynamic rehabilitation orthosis. $^{\text {? }}$
The present lower limbs rehabilitation equipment has many problems. The rehabilitation training mode is unitary. In the process of lower limbs rehabilitation training, most rehabilitation training equipment can only simulates walking posture. In most cases, the ellipse is used to replace the gait trajectory. As a result the rehabilitation training is not effective.

Aimed at the above problems, a lower limbs rehabilitation training robot adopting metamorphic theory is designed, which simulates the gait trajectory of walking and ascending steps of normal people. It can help the rehabilitation trainees to complete the rehabilitation training of two gait modes, which are walking gait and stepping gait.

Kinematics simulation and analysis of walking gait mechanism and stepping gait mechanism of lower limbs rehabilitation robot are carried out. The walking gait mechanism and stepping gait mechanism of the metamorphic lower limbs rehabilitation robot can realize the walking gait trajectory curve and stepping gait trajectory curve of normal people, so that the rehabilitation trainees can eventually achieve autonomous movements. The combination of metamorphic mechanism and lower limbs rehabilitation robot has certain significance for the research and development of lower limbs rehabilitation robot.

\section{Acquisition and analysis of normal walking and stepping gait trajectory}

Considering that most residents now live in buildings, the ultimate ideal effect for lower limbs rehabilitation training is that the trainees can walk normally and go upstairs and downstairs by themselves. The analysis to the gait of normal people plays an important role in the selection and design of mechanism of the robot, which is necessary for the design of pedal lower limbs rehabilitation robot. In the research and analysis of gait simulation and mechanism design of a lower limbs rehabilitation training robot, Liu of China University of Science and Technology collected and analyzed gait information of human walking, the results are shown in Figure $1 .{ }^{8}$

Ordinary camera is used to take videos of the tester's stepping up process, and the videos are captured frame by frame. Then, under the 
environment of MATLAB, the coordinates of the marked points are obtained by grayscale and filtering of the original photos. The curve drawn by the coordinate points got before in MATLAB is the stepping gait trajectory of normal people, which is shown in Figure 2. The blue curve represents the trajectory curve of the first metatarsal bone, and the red curve represents the trajectory curve of the heel center, and the black line between the marked points represents the posture of the tester's feet at a certain moment.

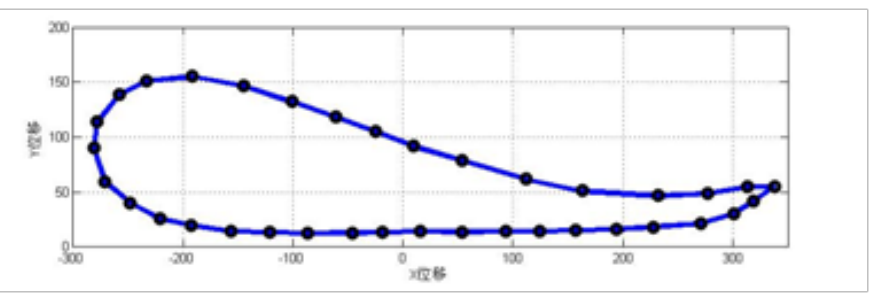

Figure I Normal gait trajectory curve.

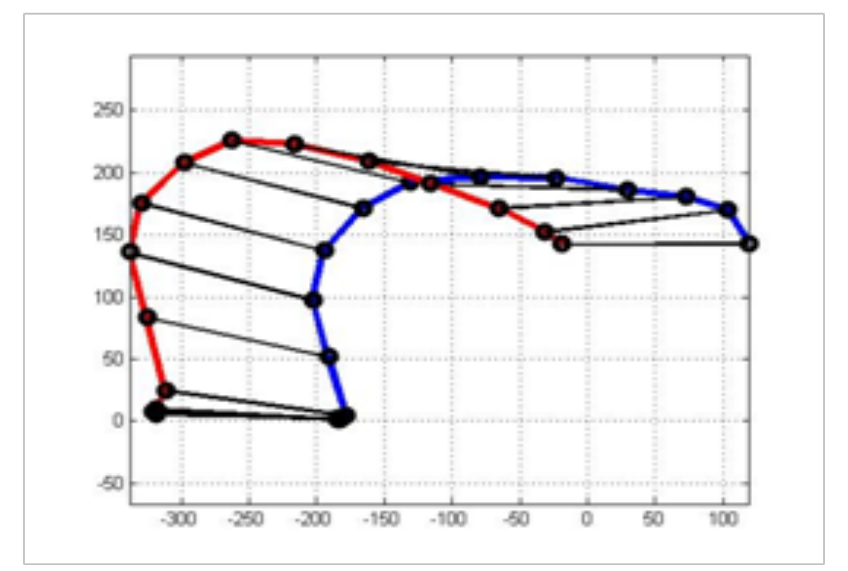

Figure 2 Stepping gait trajectory curve.

\section{Structure design of rehabilitation robots with two gait modes}

According to the aforementioned walking gait trajectory, a crankrocker mechanism is applied on walking gait rehabilitation robot. The basic parameters of the main components are obtained by referring to the linkage diagram. On this basis, the structure design of the gait mechanism is carried out. ${ }^{9,10}$

\section{Working principle of walking gait mechanism}

Figure 3 is the structure of gait rehabilitation training machine. The gait mechanism is symmetrical in structure. 7 is the frame. Servo motor 8 obtains the appropriate speed through reducer 9 . The output shaft of reducer is connected with the power input shaft of gait mechanism through coupling 10 . The power input shaft is connected with crank 11. The crank 11, connecting rod 6 , rocker 5 and frame 7 constitute the crank-rocker mechanism. The pedal 3 is connected with the connecting rod 6 . When the crank 11 rotates, the connecting rod 6 will drive the pedal 3 to produce a certain trajectory. The other end of pedal 3 is hinged with slider joint 2 . The slider joint 2 and slider 1 are fixed together. The slider 1 is reciprocating on linear track 4 , and the linear track 4 is fixed on the frame 7 according to certain requirements. When the servo motor works, the gait mechanism can simulate the heel trajectory and foot posture of the walking human. The coordinated movement of pedals on both sides is realized by controlling the rotation of the servo motors on both sides through the control system. ${ }^{11,12}$

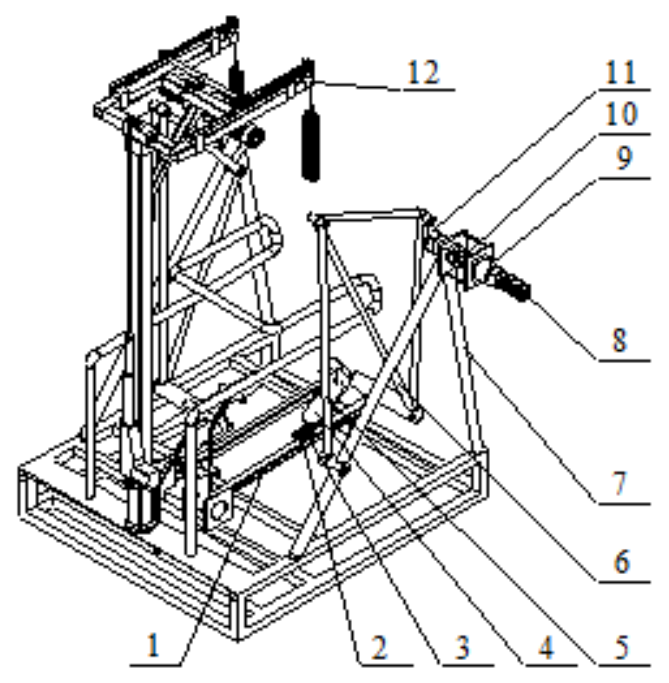

I-linear guide, 2-slider, 3-slider joint, 4-pedal, 5-rocker, 6-connecting rod, 7-bracket, 8-servo motor, 9-reducer, I0-coupling, I I-crank, I 2-weight-reducing support system

Figure 3 Mechanical structure of the robot for walking gait rehabilitation training.

\section{Working principle of stepping gait mechanism}

Figure 4 Installation process of the mechanical structure of the robot for stepping gait training

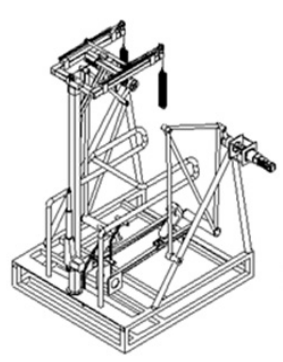

(a)

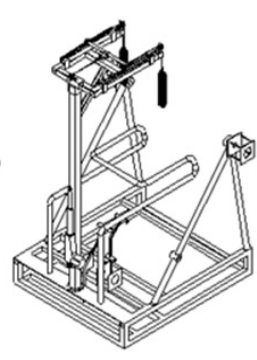

(b)

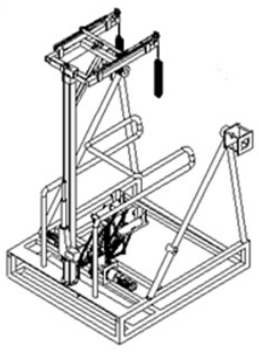

(c)
Figure 4 Installation process of the mechanical structure of the robot for stepping gait training.

When the rehabilitation achieves satisfactory results in gait rehabilitation training, the rehabilitation training on stepping gait mechanism can be carried out. Figure 4 is the refitting process of the lower limbs rehabilitation robot from a walking gait mechanism to a stepping gait mechanism. Firstly, the walking gait mechanism components, linear guide and some fittings installed on the frame are disassembled, and then the stepping gait mechanism components and their fittings are installed in corresponding positions $((a) \rightarrow(b)$ is the disassembly process, $(\mathrm{b}) \rightarrow(\mathrm{c})$ is the assembly process $).^{13,14}$

In 2.1, it is found that the shape of the two curves is basically similar by comparing the two trajectory curves. The two trajectory curves are simplified to the same trajectory curve when the stepping 
gait mechanism is designed. In the stepping gait training, it is necessary to disassemble the walking gait mechanism and linear guide rail first. And then, the stepping gait mechanism is installed.

Figure 5 is the diagram of stepping gait rehabilitation training mechanism. The stepping gait rehabilitation training mechanism is symmetrical in structure. 4 is the frame which uses the frame of the foregoing gait mechanism. 1 is the servo motor which gains the required speed for rehabilitative training through the reducer 2 . The output shaft of the reducer is connected with the power input shaft of the stepping mechanism through coupling 3.The power input shaft and the main crank 5 are coordinated by keys. There are two crankrocker mechanisms composed of crank, connecting rod, rocker and machine frame. The two crank-rocker mechanisms transmit power through connecting rod 6 . The two ends of the pedal 12 are connected to the main connecting rod 7 and the driven linkage 11 respectively. When the main crank 5 rotates, the main connecting rod will drive the pedal to produce a certain trajectory. When the servo motor works, the gait mechanism can realize the foot stepping trajectory. Finally, the coordinated movements of the pedals on both sides are realized by controlling the phase of the servo motors on both sides through the control system.

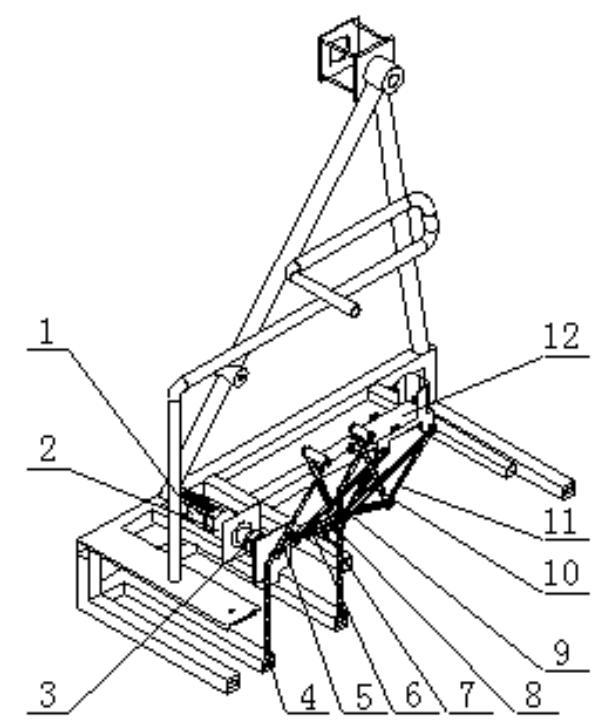

I-servo motor, 2-reducer, 3-coupling, 4-bracket, 5-main crank, 6-connecting rod, 7-main connecting rod, 8-driven crank, 9-main rocker, 10-driven rocker, I I-driven linkage, I2-pedal

Figure 5 Mechanical structure of the robot for stepping gait rehabilitation training.

\section{Kinematics simulation of rehabilitation robot with two gait modes}

\section{Kinematics simulation of stepping gait mechanism}

Working principle of stepping gait mechanism: Through disassembly and assembly, the walking gait mechanism of the metamorphic lower limbs rehabilitation robot designed can transform into stepping gait mechanism. Same as walking gait mechanism, it is also bilateral symmetry, and the two sides are driven separately to realize the foot trajectory of normal people when they go up the steps.
The kinematic diagram of stepping gait mechanism of the right side on the metamorphic lower limbs rehabilitation robot is shown in Figure 6. It can be seen that crank 1, connecting rod 2, rocker 3 and frame 4 constitute group A crank-rocker mechanism, while driven crank 8 , driven connecting rod 7, driven rocker 6 and frame 4 constitute another group B crank-rocker mechanism. Crank 1 and driven crank 8 are connected by connecting rod 9 , and the pedal 5 is connected to the connecting rod 2 and the driven connecting rod 7. The servo motor drives crank 1 to rotate in stepping gait mechanism. When crank 1 rotates to drive the crank-rocker mechanism of group A, the crank-rocker mechanism of group B rotates driven by the connecting rod 9 , so that the pedal can realize the trajectory of stepping gait rehabilitation training.

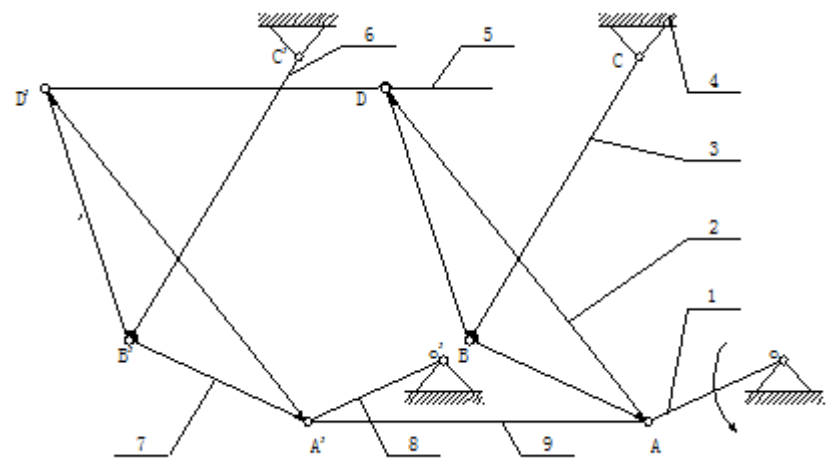

I-crank, 2-connecting rod, 3-rocker, 4-frame, 5-pedal, 6-driven rocker, 7-driven connecting rod, 8-driven crank, 9-connecting rod

Figure 6 Kinematic diagram of stepping gait mechanism.

Kinematics simulation analysis of stepping gait mechanism based on ADAMS: Similar to the walking gait mechanism, the stepping gait mechanism is also bilateral symmetry, so the kinematics analysis of the right stepping gait mechanism is carried out. The kinematic diagram of the right stepping gait mechanism is shown in Fig. 7. The coordinate system is established with $\mathrm{O}$ point as the origin. Then the vector $L_{0}=\overrightarrow{O C}, L_{1}=\overrightarrow{O A}, L_{2}=\overrightarrow{A B}, L_{3}=\overrightarrow{B C}, L_{4}=\overrightarrow{A D}$, $L_{5}=\overrightarrow{D E}, L_{6}=\overrightarrow{E F}, L_{7}=\overrightarrow{O D}, L_{8}=\overrightarrow{O F}$, are defined, and the length of vector $L_{i}$ is $l_{i}(i=0,1 \ldots 8)$ respectively. The angles of the vectors are $\theta_{i}(i=1,2 \cdots, 8)$, which are counted from the fixed axis $\mathrm{X}$ in the counterclockwise direction. The angle between $\mathrm{AD}$ and $\mathrm{AB}$ is $\alpha$, and the projection lengths of $\mathrm{OC}$ on $\mathrm{X}$ and $\mathrm{Y}$ axes are 1 and $\mathrm{h}$ respectively,

In order to verify the rationality and feasibility of the stepping mechanism of the rehabilitation robot, the size parameters of the rehabilitation robot are preliminarily determined according to the design requirements of the lower limbs rehabilitation robot and the actual situation of the rehabilitation trainees. A simplified three-dimensional model is established by Pro/E, and the motion simulation is carried out with ADAMS. Because the trajectory of the pedal determines the movement of the rehabilitation trainees during rehabilitation treatment, the pedal points $D$ and $D^{\prime}$ correspond to the position of the heel and the first metatarsal of the rehabilitation trainees, so the displacements of the point $D$ and $D^{\prime}$ on the pedals are analyzed (Figures 7-11). 


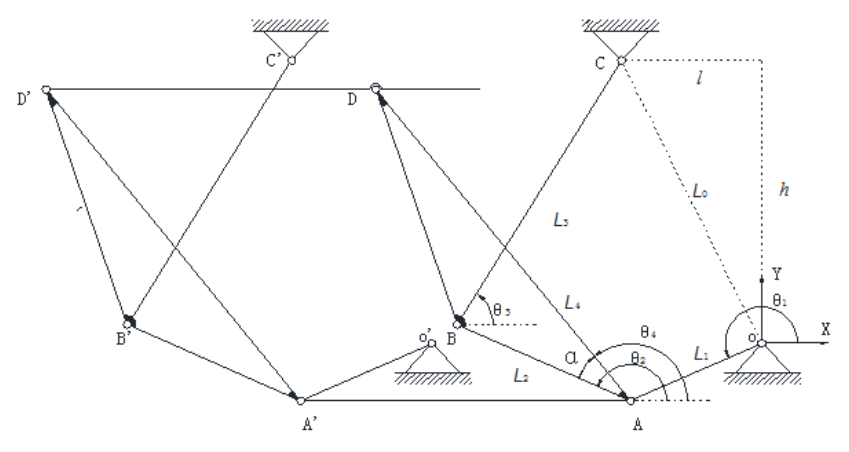

Figure 7 Kinematic diagram of the right stepping gait mechanism of the lower limbs rehabilitation robot.

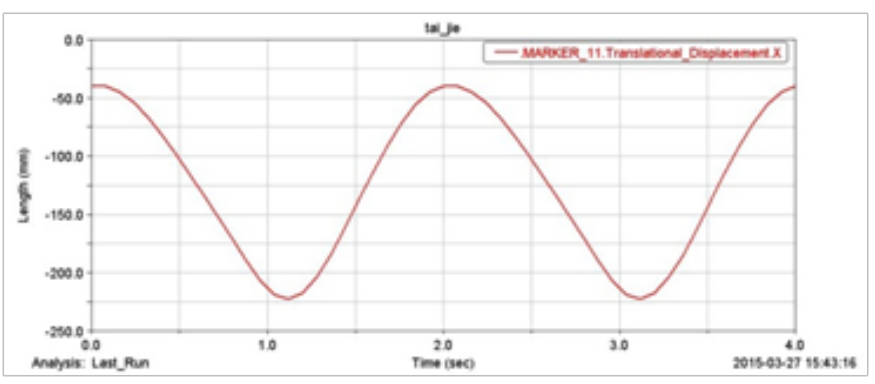

Figure 8 Displacement curve of point D over time in X-axis direction.

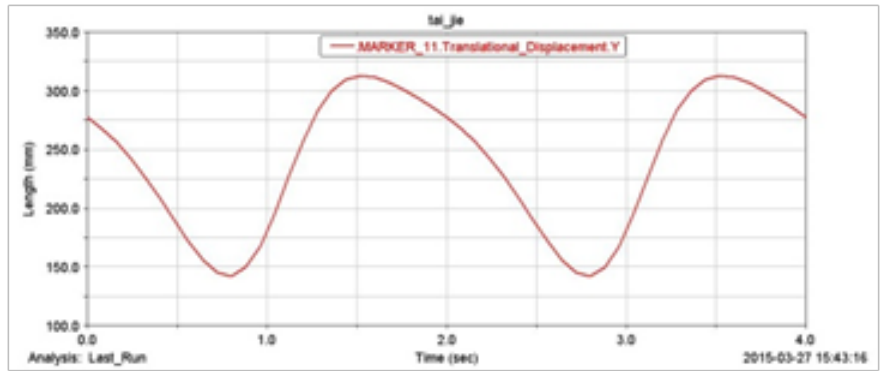

Figure 9 Displacement curve of point $D$ over time in $Y$-axis direction.

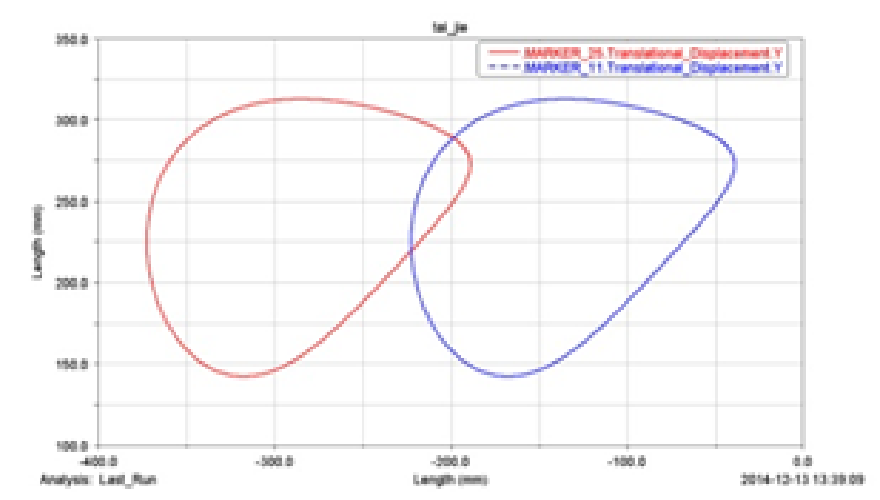

Figure 10 The trajectory curve of the two points on the pedal.

In the simulation with Adams, the angular velocity of the motor driven by the crank is, namely, a motion cycle is 2 s. The displacement curve of point $\mathrm{D}$ over time at the end of pedal in $\mathrm{X}$-axis direction is shown in Figure 8, the displacement curve of point $\mathrm{D}$ over time in Y-axis direction is shown in Figure 9, the trajectory curve of the two points $D$ and $D^{\prime}$ on pedals are shown in Figure 10, and the comparison of normal people and stepping gait mechanism trajectories is shown in Figure 11. From Figure 8, it can be seen that the displacement of point $\mathrm{D}$ over time at the end of pedal in $\mathrm{X}$ direction is a cosine curve, which is in accordance with the results obtained in the model in the previous section 4.1.The maximum displacements are $-39 \mathrm{~mm}$, and the minimum displacements are $-222 \mathrm{~mm}$, It can be concluded that the moving distance of point $\mathrm{D}$ at the end of pedal in $\mathrm{X}$ direction is $183 \mathrm{~mm}$. From Figure 9, it can be seen that the displacement of point $\mathrm{D}$ at the end of pedal in $\mathrm{Y}$ direction decreases more slowly than that of rising. The maximum displacement is $312 \mathrm{~mm}$, and the minimum displacement is $142 \mathrm{~mm}$. It can be concluded that the distance of point $\mathrm{D}$ at the end of pedal in $\mathrm{Y}$ direction is $170 \mathrm{~mm}$ in a cycle.

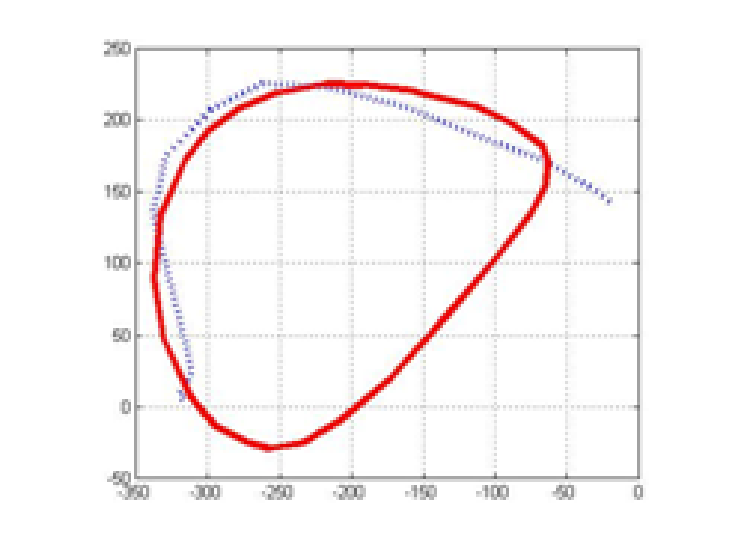

Figure II Comparison of normal people and stepping gait mechanism trajectories (units:mm).

The space trajectory of the two points $D$ and $D^{\prime}$ on pedals during walking rehabilitation on stepping gait mechanism has been displayed in Figure 10. The two points are corresponding to the position of the heel center and the first metatarsal of the rehabilitation trainees. Through the previous analysis, it is known that the movement curve of the heel center is similar to that of first the metatarsal in the stepping up process. Therefore, the curve of the first metatarsal is analyzed here to achieve very good correction effect. In Figure 11, the curve represented by the blue dotted line is the stepping up trajectory curve of the first metatarsal of the normal people, while the red curve represents the gait trajectory of the same position in the stepping gait mechanism of the lower limbs rehabilitation robot. The red curve is a closed curve, because there will be a reset process during the stepping gait rehabilitation training. Through the comparison of the two curves, it is found that there are bits of differences between the trajectory of stepping up gait mechanism and that of normal people. But the changing trends of the two trajectory are basically the same, so that the rehabilitation exercise can be achieved.

Similarly, kinematics simulation of walking gait mechanism can also be obtained by using the above methods. By comparing the trajectory of walking gait mechanism with that of normal people, it is found that there are bits of differences between the trajectory of walking gait mechanism and that of normal people. But the changing trend of trajectory is basically the same, and the rehabilitation exercise can be achieved. 


\section{Conclusion}

A lower limbs rehabilitation robot with metamorphic function is designed based on metamorphic mechanism theory, which can implement walking and stepping gait rehabilitation training, and can adapt to the walking and stepping gait rehabilitation training. It provides an example for the practical application of metamorphic mechanism theory because of its self-adaptability and reconfigurability. Similarly, kinematics simulation of walking gait mechanism can also be carried out by using the above methods. By comparing the trajectories of walking gait mechanism and that of normal people, it is found that there are some differences between the trajectories of walking gait mechanism and that of normal people. However, the trend of movement track is basically the same, and rehabilitation exercise can be achieved.

\section{Acknowledgments}

None.

\section{Conflicts of interest}

The author declares there is no conflict of interest.

\section{References}

1. Schmidt Henning, Cordula Werner, Rolf Bernhardt, et al. Gait rehabilitation machines based on programmable footplates. Journal of NeuroEngineering and Rehabilitation. 2007;4:2.

2. Fisher S, Lucas L, Thrasher TA. Robot-Assisted Gait Training for Patients with Hemiparesis Due to Stroke. Topics in Stroke Rehabilitation. 2011;18(3):269-276.

3. Surdilovic Dragoljub, Rolf Bernhardt, Tobias Schmidt, et al. 26 STRINGMEN: A Novel Wire Robot for Gait Rehabilitation. Advances in Rehabilitation Robotics. 2004. p. 413-424.
4. Shiozawa N, Arima S, Makikawa M. Virtual walkway system and prediction of gait mode transition for the control of the gait simulator. Conf Proc IEEE Eng Med Biol Soc. 2004;4: 2699-2702.

5. Hesse S, Uhlenbrock D. A mechanized gait trainer for restoration of gait. Journal of Rehabilitation Research \& Development. 200;37(6):701-708.

6. Nelson Costa, Milan Bezdicek, Michael Brown, et al. Joint motion control of a powered lower limbs orthosis for rehabilitation. International Journal of Automation and Computing. 2006;3(3):271-281.

7. Liu XH. Application of lower limbs orthosis in paralysis rehabilitation. Journal of Clinical Rehabilitative Tissue Engineering Research. 2007.

8. Liu YY. Gait Simulation and Mechanism design Analysis of Lower limbs Rehabilitation Training Robot Diss. 2013.

9. Colby SM, Kirkendall DT, Bruzga RF. Electromyographic analysis and energy expenditure of harness supported treadmill walking: implications for knee rehabilitation. Gait \& Posture. 1999;10(3):200-205.

10. Dangxiao Wang, Jiting Li, Chao Li, et al. An adaptive haptic interaction architecture for knee rehabilitation robot. International Conference on Mechatronics \& Automation IEEE. 2009.

11. Lim HB, Hoon Hk, Low KH, et al. Pelvic control and over-ground walking methodology for impaired gait recovery. IEEE International Conference on Robotics \& Biomimetics. 2009.

12. Fengfeng $\mathrm{Xi}$, Younan $\mathrm{Xu}$, Guoliang Xiong. Design and analysis of a re-configurable parallel robot. Mechanism \& Machine Theory. 2006;41(2):191-211.

13. Xianwen Kong, Clément M Gosselin, Pierre-Luc Richard, et al. Type Synthesis of Parallel Mechanisms with Multiple Operation Modes. J Mech Des. 2007;129(6):595-601.

14. Carbonari L, Callegari M, Palmieri G, et al. A new class of reconfigurable parallel kinematic machines. Mechanism and Machine Theory. 2014;79:173-183. 